\title{
Internet-delivered therapy for anxiety disorders: a solution to unequal access to treatment? ${ }^{\dagger}$ COMMENTARY ON... COCHRANE CORNER
}

\author{
Mary Jane Attenburrow \& Katharine Smith
}

\begin{abstract}
SUMMARY
Anxiety disorders are common, often have a chronic course and frequently coexist with other psychiatric disorders. Psychological therapy is recommended as first-line treatment, but equitable access remains a challenge. This month's Cochrane Corner review assesses the evidence for the efficacy of therapist-supported cognitive-behavioural therapy (CBT) for anxiety disorders delivered via the internet. Although internet delivery of therapy is attractive for many reasons, and the results of this preliminary review suggest that it is efficacious, this is a rapidly expanding field. Further updates of this review will include more evidence to support or refute the use of this new method of treatment delivery, either alongside or in preference to standard faceto-face CBT.
\end{abstract}

\section{DECLARATION OF INTEREST}

None

\section{Clinical background}

Symptoms of anxiety are common in the general population, and in primary and secondary care. In the general population, the 12-month period prevalence for all anxiety disorders is about $14 \%$ and the lifetime prevalence about $21 \%$. Individual disorders have an estimated 12-month prevalence rate ranging from $0.7 \%$ (obsessive-compulsive disorder, OCD) to $6.4 \%$ (specific phobia), and an estimated lifetime prevalence of $0.8-13.2 \%$ (Wittchen 2005, 2011). Anxiety disorders are a major contributor to the global burden of disease (Vos 2012). They tend to have an onset in childhood or adolescence (Kessler 2005) and a chronic course. There are high levels of comorbidity between anxiety disorders and bipolar disorder, major depression, substance misuse, schizophrenia and physical illness. Comorbidity worsens the outcome and presents more of a treatment challenge (Baldwin 2014).
First-line treatment for anxiety disorders focuses on psychological therapies. However, despite the evidence, it is well recognised that there is variable provision of services from diagnosis to treatment for these disorders. To address this disparity, the National Institute for Health and Care Excellence (NICE) issued a quality standard (Box 1) for anxiety disorders (QS53; NICE 2014). This recognises that, despite recommendations, pharmacological treatment is frequently used first line, perhaps in part because access to evidence-based psychological therapies is variable. The quality standard recommends that the treatment of anxiety disorders in the UK is based on a stepped-care approach (NICE 2011), with cognitive-behavioural therapy (CBT) recommended as one of the first-line treatments for generalised anxiety disorder, panic disorder, posttraumatic stress disorder (PTSD), OCD and body

\section{ROUND THE} CORNER
Mary Jane Attenburrow is Clinical Lead at the National Institute for Health Research (NIHR) Oxford cognitive health Clinical Research Facility, a Senior Research Fellow at Oxford University Department of Psychiatry and an honorary consultant psychiatrist. Katharine Smith is an honorary consultant psychiatrist at the NIHR Oxford cognitive health Clinical Research Facility and Oxford University Department of Psychiatry. She has dual accreditation as a consultant in both general adult and old age psychiatry.

Correspondence Dr Mary Jane Attenburrow, Department of Psychiatry, Warneford Hospital, Oxford OX3 7JX, UK. Email: maryjane.attenburrow@psych.ox.ac.uk

${ }^{\dagger}$ See p. 290, this issue.
BOX 1 What are NICE quality standards?

- Concise sets of prioritised statements designed to drive measurable quality improvements within a particular area of health or social care

- Derived from the best available evidence, such as NICE guidance and other evidence sources accredited by NICE

- Developed independently by NICE, in collaboration with health and social care professionals, their partners and patients

- Audiences include health commissioners, service delivery staff in primary and secondary care, patients and carers

- The standards help stakeholders to assess care expectations, evaluate delivery and measure activity or output (the measurement in QS53 on anxiety disorders is 'the proportion of people with an anxiety disorder who receive evidence-based psychological treatment intervention' (NICE 2014)) 
dysmorphic disorder. For social anxiety disorder, patients should have access to treatment with CBT specifically developed for this purpose (NICE 2013), and for PTSD, access to trauma-focused CBT (NICE 2005).

To enable consistent access to psychological therapies in the National Health Service (NHS), the Improving Access to Psychological Therapies (IAPT) programme was launched in 2008 (Department of Health 2008), with significant government financial investment. IAPT was set up to train psychological therapists to deliver therapies with a focus on CBT, with the roll-out programme completing in 2015 and aiming by the end of 2015 to see 900000 patients a year. The programme was on target when reported in 2011, but with variable equity of access and recovery rates depending on the site (Clark 2011).

Individuals are increasingly making use of online resources to support their own health and knowledge of treatment. In the UK, 83\% of homes now have internet access and $43 \%$ of internet users report having used the web to access health information, compared with 18\% in 2007 (Cotton 2014). In the context of this rapidly expanding field, the recent launch of ResearchKit by Apple (an open source software platform allowing researchers to develop apps for healthcare research and delivery: www.apple.com/uk/researchkit/) attracted worldwide attention.

Internet delivery of CBT has the potential to minimise treatment barriers, increase access to care and be more efficient in terms of cost and speed of dissemination. Evidence suggests that internetbased psychological treatments are more effective with regular support from a clinician (Palmqvist 2007), but the total amount of therapist time is much less than in face-to-face therapy.

Initial reviews of internet-based treatments for mental health problems have suggested that these are more effective than a waiting-list control and may be as effective as face-to-face therapy in treating anxiety and depression (e.g. Cuijpers 2009). This month's Cochrane Corner review (Olthuis 2015; for abstract see pp. 290, this issue) focused on therapist-supported internet-delivered CBT (ICBT) for anxiety disorders.

\section{Main results}

For included studies, the risk of bias was assessed independently by two authors and study quality was judged. Wherever possible, data were used from intention-to-treat analysis (Box 2).

Treatment effect where the outcome was dichotomous was assessed using a risk ratio (RR)
BOX 2 Intention-to-treat analysis

The results of randomised controlled trials (RCTs) can be biased if the data analysed are from comparison groups that differ from the groups originally generated by randomisation. This might be because participants drop out of the study, do not adhere fully to the therapy or are switched to alternative therapies. Effect estimates can be biased if these data are either left out of the analysis or allocated to a different comparison group. For example, an efficacy estimate might be inflated or adverse effects not accounted for. Intention-to-treat (ITT) is the least biased way to estimate intervention effects in RCTs.

The principles in ITT analysis are:

- participants are kept in the groups to which they were randomised, regardless of the intervention they actually received

- outcome data are measured for all participants

- all randomised participants are included in the analysis. In practice, RCTs are very likely to have missing outcome data, especially if there is a long follow-up period. However, this can be managed by means of a number of techniques, such 'last observation carried forward' (LOCF) and using assumed outcomes. Missing data might be replaced with an assumed poor outcome, a mean value or one predicted by a regression analysis.

(Higgins 2011)

(Box 3) with a 95\% confidence interval. Outcomes that were based on continuous scores were assessed using standardised mean differences (SMD) (Box 4).

Thirty trials ( $n=2181$ participants) were included in the review, which considered the efficacy of therapist-supported ICBT in comparison

\section{BOX 3 Risk ratio or relative risk}

The risk ratio or relative risk (RR) is a measure of the outcome in one group relative to that in the other. Specifically, it is the ratio of the risk of an event in the two groups, where an 'event' is defined by the outcome measure and 'risk' is the number of participants with the event divided by the total number of participants in that group. The risk in one group divided by risk in another group is the relative risk:

\section{risk of event in experimental group

$$
\text { risk of event in control group }
$$

A value of 1.0 indicates there is no difference between the interventions.

RR describes the multiplication of the risk that occurs with the intervention in the experimental group, therefore an RR of 2.0 implies that an event is twice as likely with the intervention than without. 


\section{BOX 4 Standardised mean difference}

The standardised mean difference (SMD) is used as a summary statistic in meta-analysis when the studies being examined all assess the same outcome but measure it in a variety of ways. For example, the studies in Olthuis et al's review used different measures of disorder-specific anxiety and quality of life. The results therefore needed to be standardised before they could be combined.

The SMD expresses the size of the intervention effect in each study relative to the variability observed in that study. The intervention effect is therefore a difference in means, and not a mean of differences. The SMD is calcuated as:

\section{difference in mean outcome between groups}

standard deviation of outcome among participants

Directionality is important in calculating the SMD, and must also be taken into account when interpreting results. Thus, both a positive and a negative SMD can indicate an effect in favour of an intervention, depending on which of an increasing or decreasing value of the test score in question represents an improvement. Clearly, a pooled estimate of SMDs of zero in a meta-analysis means that there is no difference between intervention and control.

with three interventions - a waiting-list control, traditional face-to-face CBT, and self-help - in treating anxiety disorders in adults.

The results from studies that gave moderatequality evidence showed that therapist-supported ICBT is more efficacious than a waiting-list, attention, information or online discussion group only control in leading to clinically important improvement in anxiety (determined by a diagnostic interview such as the Structured Clinical Interview for DSM Disorders (SCID) or by a defined cut-off on a validated scale such as the Clinical Global Impressions (CGI) scale) ( $n=644$, $\mathrm{RR}=4.18,95 \%$ CI 2.42-7.22).

In addition, compared with face-to-face CBT, therapist-supported ICBT showed no significant differences post-treatment in clinically important improvement in anxiety $(n=365$; RR $=1.09,95 \%$ CI 0.89-1.34) or in disorder-specific and general anxiety symptoms. Other reported results were based on low- or very low-quality evidence or the numbers of participants in the studies were too small to make meaningful comparisons.

\section{Discussion}

The main findings suggest that therapist-supported ICBT may be a valid form of treatment delivery for anxiety disorders. This is important as ICBT could improve access to treatment and be more cost-effective, particularly in rural communities. Face-to-face CBT is currently the intervention of choice for the treatment of anxiety disorders (NICE 2014), but access to psychological therapies despite IAPT remains a challenge, and the review suggests that therapist-supported ICBT may be an effective alternative.

\section{Generalisability and consistency of findings}

Although the main findings of this review suggest that therapist supported ICBT is more efficacious than a number of comparator conditions and is similar to face-to-face delivery, caution should be exercised when assessing the generalisability of the results because of the degree of heterogeneity in the data. Olthuis et al suggest that this may be related to the different types of anxiety disorder studied and the variability in the exact details of the interventions in the included studies. Separating the data into subgroups for separate analysis produced results that were difficult to interpret because the number of studies in each group was low.

The main results are generally consistent with those of a previous Cochrane review (Mayo-Wilson 2013) on media-delivered CBT and behavioural therapy, with and without therapist contact, for anxiety disorders. That review also suggested that ICBT was more effective than a waiting-list control. Although, unlike the current review, the results suggested that ICBT was inferior to face-to-face CBT, this difference may have been accounted for by the inclusion of ICBT without therapist support.

\section{Clinical applicability}

The applicability of Olthuis et al's findings to clinical practice is potentially limited by several factors.

First, the majority of studies included in the review focused on panic disorder, social phobia and generalised anxiety disorder. More research is needed into ICBT for other anxiety disorders, such as OCD, PTSD and specific phobia. Treatments for these disorders rely heavily on exposure-based elements, which may have deterred researchers from translating them into an ICBT intervention.

Second, the interventions varied in length and content and need to be standardised for a reliable comparison across studies.

Third, the majority of the studies recruited participants through media adverts. It is typical for ICBT trials to recruit through the media, whereas RCTs of face-to-face CBT often recruit 
via secondary care clinic referral. This raises concerns as to whether the two populations differ, especially with regard to severity of symptoms. However, a study by Titov et al (2010) found that symptom severity, distress and disability in ICBT participants were as severe as in individuals attending a face-to-face clinic.

Finally, in secondary care services, anxiety disorders are commonly comorbid with other axis I disorders. Although most of the included trials recruited some participants with comorbid conditions, this was not the focus of the review and the analyses were not able to address whether comorbidity (or indeed concomitant treatment with medication) had an effect on outcome.

\section{The future}

The use of the internet to deliver specific psychological treatments is a rapidly expanding area that needs high-quality research and assessment. In Olthuis et al's review, the results were based on an initial search performed in April 2013. An updated search only 18 months later (in September 2014) identified four new completed studies, seven previously ongoing studies completed and three new ongoing studies, all of which will be included in the next update of the review. This update will give a larger evidence base on which to draw further conclusions regarding the efficacy of ICBT and, more specifically, its direct comparison with face-to-face treatment.

\section{Acknowledgements}

The authors acknowledge support from the NIHR Oxford cognitive health Clinical Research Facility. The views expressed are those of the authors and not necessarily those of the NHS, the NIHR or the Department of Health.

\section{References}

Baldwin DS, Anderson IM, Nutt DJ, et al (2014) Evidence-based pharmacological treatment of anxiety disorders, post-traumatic stress disorder and obsessive-compulsive disorder: a revision of the 2005 guidelines from the British Association for Psychopharmacology. Journal of Psychopharmacology, 28: 403-39.
Clark M (2011) Implementing NICE guidelines for the psychological treatment of depression and anxiety disorders: the IAPT experience. International Review of Psychiatry, 23: 318-27.

Cotton R, Irwin J, Wilkins A, et al (2014) The Future's Digital: Mental Health and Technology. Mental Health Network NHS Confederation.

Cuijpers P, Marks IM, van Straten A, et al (2009) Computer-aided psychotherapy for anxiety disorders: a meta-analytic review. Cognitive Behaviour Therapy, 38: 66-82.

Department of Health (2008) Improving Access to Psychological Therapies Implementation Plan: National Guidelines for Regional Delivery. Department of Health.

Higgins JPT, Green S (eds) (2011) Cochrane Handbook for Systematic Reviews of Interventions Version 5.1.0 (Updated March 2011). Cochrane Collaboration.

Kessler RC, Berglund P, Demler O, et al (2005) Lifetime prevalence and age-of-onset distributions of DSM-IV disorders in the national comorbidity survey replication. Archives of General Psychiatry, 62: 593-602.

Mayo-Wilson E, Montgomery P (2013) Media-delivered cognitive behavioural therapy and behavioural therapy (self-help) for anxiety disorders in adults. Cochrane Database of Systematic Reviews, 9: doi: 10.1002/14651858.CD005330.pub4.

National Institute for Health and Care Excellence (2005) Post-Traumatic Stress Disorder (PTSD): The Management of PTSD in Adults and Children in Primary and Secondary Care (NICE Clinical Guideline CG26). NICE.

National Institute for Health and Care Excellence (2011) Common Mental Health Disorders: Identification and Pathways to Care (NICE Clinical Guideline CG123). NICE.

National Institute for Health and Care Excellence (2013) Social Anxiety Disorder: Recognition, Assessment and Treatment (NICE Clinical Guideline CG159). NICE.

National Institute for Health and Care Excellence (2014) Anxiety Disorders (NICE Quality Standard OS53). NICE.

Olthuis JV, Watt MC, Bailey K, et al (2015) Therapist-supported Internet cognitive behavioural therapy for anxiety disorders in adults. Cochrane Database of Systematic Reviews, 3: doi: 10.1002/14651858.CD011565.

Palmqvist B, Carlbring P, Andersson G (2007) Internet-delivered treatments with or without therapist input. Expert Review of Pharmacoeconomics Outcomes Research, 7: 291-7.

Titov N, Andrews G, Kemp A, et al (2010) Characteristics of adults with anxiety or depression treated at an internet clinic: comparison with a national survey and an outpatient clinic. PLOS ONE, 5: e10885.

Vos T, Flaxman AD, Naghavi M, et al (2012) Years lived with disability (YLDs) for 1160 sequelae of 289 diseases and injuries 1990-2010: a systematic analysis for the Global Burden of Disease Study. Lancet, 380: 2163-96.

Wittchen HU, Jacobi F (2005) Size and burden of mental disorders in Europe: a critical review and appraisal of 27 studies. European Neuropsychopharmacology, 15: 357-76.

Wittchen HU, Jacobi F, Rehm J, et al (2011) The size and burden of mental disorders and other disorders of the brain in Europe. European Neuropsychopharmacology, 21: 655-79. 